\title{
FAST CONVERGENCE OF THE COIFLET-GALERKIN METHOD FOR GENERAL ELLIPTIC BVPS
}

\author{
HANI AKBARI \\ Department of Applied Mathematics \\ Ferdowsi University of Mashhad, Mashhad, Iran \\ e-mail: haniakbari@gmail.com
}

\begin{abstract}
We consider a general elliptic Robin boundary value problem. Using orthogonal Coifman wavelets (Coiflets) as basis functions in the Galerkin method, we prove that the rate of convergence of an approximate solution to the exact one is $O\left(2^{-n N}\right)$ in the $H^{1}$ norm, where $n$ is the level of approximation and $N$ is the Coiflet degree. The Galerkin method needs to evaluate a lot of complicated integrals. We present a structured approach for fast and effective evaluation of these integrals via trivariate connection coefficients. Due to the fast convergence rate, very good approximations are found at low levels and with low Coiflet degrees, hence the size of corresponding linear systems is small. Numerical experiments confirm these claims.
\end{abstract}

Keywords: general stationary elliptic BVPs, Robin boundary condition, orthogonal Coifman wavelets, trivariate connection coefficients, fast convergence.

\section{Introduction}

Let $\Omega$ be a bounded open set in $\mathbb{R}^{d}$ and $\partial \Omega$ its Lipschitz boundary. The outward normal of $\Omega$ is denoted by $\mathbf{n}$. Consider two differential operators $\mathcal{L}, \mathcal{B}$ in the forms

$$
\begin{aligned}
& \mathcal{L} u=-\nabla \cdot(\sigma \cdot \nabla u)+\beta \cdot \nabla u+\mu u, \\
& \mathcal{B} u=\mathbf{n} \cdot \sigma \cdot \nabla u+\gamma u,
\end{aligned}
$$

where $\sigma, \beta$ and $\mu$ are functions defined over $\Omega$ and taking their values in $\mathbb{R}^{d, d}, \mathbb{R}^{d}$ and $\mathbb{R}$, respectively, and $\gamma$ is a real valued function defined over $\partial \Omega$.

Given two functions $f: \Omega \rightarrow \mathbb{R}$ and $g: \partial \Omega \rightarrow \mathbb{R}$, consider the problem of finding a function $u: \Omega \rightarrow \mathbb{R}$ such that

$$
\begin{cases}\mathcal{L}(u)=f & \text { in } \Omega \\ \mathcal{B}(u)=g & \text { on } \partial \Omega\end{cases}
$$

The boundary condition in the problem (3) is called the third type or the Robin boundary condition. This equation is applicable in many fields of physics and engineering, e.g., heat transfer with a convection boundary or advection diffusion problems.

The Galerkin method is a common technique used for numerical solution of differential and integral equations (Bandrowski et al., 2010). In brief, it converts the boundary value problem to a variational formulation.
Considering a family of basis functions, the variational formulation is converted to a linear system where its corresponding matrix is called the stiffness matrix. The unknowns of the linear system are expansion coefficients of an approximate solution in linear combination of basis functions.

A common choice of basis functions are finite element interpolation functions (Reddy, 2006; Ern and Guermond, 2004), but application of wavelets in the Galerkin method (for differential and integral equations) is too wide (Akbari and Kerayechian, 2012; Saberi et al., 2012; El-Gamel, 2006; Hashish et al., 2009; Nowak et al., 2010; Vampa et al., 2010).

Nevertheless, general problems like (3) are rarely considered. These kinds of problems call for computing a lot of complicated integrals, called 'connection coefficients', to obtain the linear system; especially when the variational formulation is not symmetric, we have to evaluate all entries of the stiffness matrix, not nearly half the total entries in a symmetric situation. Moreover, the arising linear systems are ill-conditioned and therefore the solutions are not stable. Hence the theory does not coincide with numerical experiments. Nearly all papers in this area contain several references to preconditioning techniques to solve ill-conditioned linear systems. As has been mentioned, we use Coiflets as basis functions 
in the Galerkin method, i.e., for $\varphi$, a Coiflet of degree $N$, and in the level of approximation $n$, we consider the family of $\left\{\varphi\left(2^{n} x-i\right)\right\}$ as basis functions. The sampling approximation theorem shows that Coiflets are powerful in interpolating functions (see Theorem 1). With the help of this theorem, we find a suitable approximation of variational formulation which facilitates the proof of the fast rate of convergence and especially fast evaluation of stiffness matrix entries.

The fast rate of convergence results in very good numerical results, at low levels of approximation, with low degrees of Coiflets, because the size of the corresponding linear system becomes (relatively) small and we can use regularization techniques to solve linear systems (Jensen and Hansen, 2007). These techniques apply (somehow) dense factorizations of the stiffness matrix which is not suitable for large scale systems, in contrast to preconditioning methods, where sparse (usually deflected) factorizations are considered (Saad, 1996). Instead, regularization techniques are more accurate and therefore we find stabilized solutions to ill-conditioned linear systems.

Accurate and fast evaluation of stiffness matrix entries depends on accurate and fast evaluation of connection coefficients. A problem that arises in the wavelet-Galerkin method is evaluation of these values on the interval (Lin and Zhou, 2001). The highly oscillatory nature of wavelet basis functions makes standard numerical quadrature of integrals near the boundary impractical. This difficulty motivates the researchers to combine the wavelet-Galerkin method with the fictitious domain approach. As a result, evaluation of connection coefficients on the interval converts to evaluation on $\mathbb{R}^{d}$, which is a simple and straightforward process. Moreover, domains with an arbitrary boundary can be considered, but some additional computations are imposed (Baccou and Liandrat, 2006; Resnikoff and Wells, 1998).

With a simple and effective approach, we precisely evaluate connection coefficients on the interval. Exact values of Coiflet coefficients, presented by Cerna et al. (2008), help us to increase the accuracy. With this facility we do not use the fictitious domain approach and consequently additional computations are discarded.

Outline. First we give an introduction to Coiflets and the sampling approximation theorem. In Section 3 , the approximation of variational formulation is proposed and we prove fast convergence with the rate of $O\left(2^{-n N}\right)$. Next we define and evaluate trivariate connection coefficients and we use them to implement the Coiflet-Galerkin method. Finally, to confirm the theory and effectiveness of the proposed method, some numerical experiments are presented.

\section{Coiflets}

In 1989, R. Coifman suggested the design of orthogonal wavelet systems with vanishing moments for both scaling and wavelet functions. They were first constructed by Daubechies (1992), who named them Coiflets. The construction of Coiflets and their properties are treated, e.g., by Cerna et al. (2008), Daubechies (1992), or Resnikoff and Wells (1998). This section is a summary of the work of Resnikoff and Wells (1998, Section 9.2.).

Let $\varphi, \psi$ be, respectively, the scaling and the wavelet functions, where $\varphi$ satisfies the refinable equation,

$$
\varphi(x)=\sum_{k=N_{0}}^{N_{1}} a_{k} \varphi(2 x-k) .
$$

The compact support of $\varphi$ is $\left[N_{0}, N_{1}\right]$.

$$
\text { Let } \varphi_{k}(x)=\varphi(x-k) \text { and } \varphi_{n k}(x)=2^{\frac{n}{2}} \varphi_{k}\left(2^{n} x\right) \text {. }
$$

Definition 1. An orthonormal wavelet system with compact support is called a Coiflet system of degree $N$ if the functions $\varphi$ and $\psi$ satisfy

$$
\begin{aligned}
\int x^{l} \psi(x) \mathrm{d} x=0, \quad \int x^{l} \varphi(x) \mathrm{d} x & =\delta_{0 l}, \\
l & =0, \ldots, N .
\end{aligned}
$$

Lemma 1. Let $\varphi$ be a continuous Coiflet scaling function of degree N. Then

$$
\sum_{k}(x-k)^{l} \varphi_{k}(x)=\delta_{0 l}, \quad l=0, \ldots, N
$$

Moreover, if $\varphi$ is differentiable, then

$$
\sum_{k}(x-k)^{l} \varphi_{k}^{\prime}(x)=-\delta_{1 l}, \quad l=0, \ldots, N .
$$

Remark 1. Henceforth, $c$ is constant, independent of $n$, and not necessarily the same at each occurrence.

For Coiflets, the sampling approximation theorem asserts the exponential approximation in the degree, by sampling at equidistant points.

Theorem 1. (Sampling approximation theorem) Let $\Omega$ be a domain in $\mathbb{R}^{d}$ and $\varphi \in C^{\alpha}(\mathbb{R})$ a differentiable $(\alpha \geq 1)$ Coiflet scaling function of degree N. Let

$$
\begin{gathered}
X=\left(x_{1}, \ldots, x_{d}\right) \in \mathbb{R}^{d}, \quad I=\left(i_{1}, \ldots, i_{d}\right) \in \mathbb{Z}^{d}, \\
\Phi_{I}^{n}(X)=\varphi_{n i_{1}}\left(x_{1}\right) \cdots \varphi_{n i_{d}}\left(x_{d}\right) . \\
\text { If } f \in C^{N, 1}(\bar{\Omega}), \text { then } \\
\left\|f-S^{n} f\right\|_{L^{2}(\Omega)} \leq c 2^{-n(N+1)}, \\
\left\|f-S^{n} f\right\|_{H^{1}(\Omega)} \leq c 2^{-n N},
\end{gathered}
$$


where

$$
S^{n} f(X)=2^{-\frac{d}{2} n} \sum_{I \in \Lambda_{n}} f\left(\frac{i_{1}}{2^{n}}, \ldots, \frac{i_{d}}{2^{n}}\right) \Phi_{I}^{n}(X) .
$$

The index set $\Lambda_{n}$ is

$$
\Lambda^{n}=\left\{I \in \mathbb{Z}^{d} \mid \operatorname{supp}\left(\Phi_{I}^{n}(X)\right) \cap \Omega \neq \emptyset\right\} .
$$

The constant $c$ depends on $\varphi$ and the smoothness off.

Henceforth, when we write $S^{n} f$, the hypothesis of Theorem 1 is assumed implicitly. A part of the proof of Theorem 1 is adopted to prove what follows.

Proposition 1. Let $f \geq \bar{\delta}>0$ for a constant $\bar{\delta}$. There exist $n \geq 0$ and a positive constant $\delta$ such that $S^{n} f \geq \delta>0$.

Here $\alpha$ in $\varphi \in C^{\alpha}(\mathbb{R})$ is called a Sobolev exponent and shows the smoothness of scaling function. For a fixed Coiflet degree, different scaling coefficients, $a_{k} \mathrm{~s}$, can be found (Resnikoff and Wells, 1998), but they differ in $\alpha$. The scaling coefficients and the Sobolev exponent of our desired Coiflets are presented in Table 1. They are obtained from the method proposed by Cerna et al. (2008).

\section{Coiflet-Galerkin method and error analysis}

In this section we prove the fast rate of convergence. Henceforth, the following assumptions are made on the data of Eqn. (3) to confirm the existence of integrals in variational formulation and its approximation:

$$
\begin{aligned}
\sigma & \in\left[L^{\infty}(\Omega)\right]^{d, d}, & \beta & \in\left[L^{\infty}(\Omega)\right]^{d}, \\
\gamma & \in L^{\infty}(\partial \Omega), & \mu, \nabla \cdot \beta & \in L^{\infty}(\Omega), \\
f & \in L^{2}(\Omega), & g & \in L^{2}(\partial \Omega),
\end{aligned}
$$

Moreover, we consider the operator $\mathcal{L}$ in (1) to be elliptic i.e., a constant $\sigma_{0}>0$ exists such that for all $\xi \in$ $\mathbb{R}^{d}$ the matrix $\sigma$ satisfies

$$
\xi \cdot \sigma(X) \cdot \xi=\sum_{i, j=1}^{d} \sigma_{i j} \xi_{i} \xi_{j} \geq \sigma_{0}\|\xi\|_{2}^{2} \quad \text { a.e. in } \Omega \text {. }
$$

Multiplying $\mathcal{L}(u)=f$ by a (sufficiently smooth) test function $v$, integrating over $\Omega$, using Green's formula, and applying $\mathcal{B}(u)=g$, we obtain the weak formulation,

$$
\begin{aligned}
\int_{\Omega} \nabla v \cdot \sigma \cdot \nabla u & +\int_{\Omega}(\beta \cdot \nabla u) v \\
& +\int_{\Omega} \mu u v+\oint_{\partial \Omega} \gamma u v=\int_{\Omega} f v+\oint_{\partial \Omega} g v .
\end{aligned}
$$

Note that $u, v \in H^{1}(\Omega)$ is a possible regularity requirement on $u$ and $v$ for the integrals over $\Omega$ to be meaningful (Ern and Guermond, 2004). Hence we consider the following problem:

$$
\left\{\begin{array}{l}
\text { Find } u \in H^{1}(\Omega), \quad \text { s.t. } \\
a(u, v)=\ell(v), \quad \forall v \in H^{1}(\Omega),
\end{array}\right.
$$

where the bilinear form $a$ and the linear form $l$ are

$$
\begin{aligned}
a(u, v)= & \int_{\Omega} \nabla v \cdot \sigma \cdot \nabla u \\
& +\int_{\Omega}(\beta \cdot \nabla u) v+\int_{\Omega} \mu u v+\oint_{\partial \Omega} \gamma u v, \\
l(v)= & \int_{\Omega} f v+\oint_{\partial \Omega} g v .
\end{aligned}
$$

The relation of the problems (3) and (13) and properties of bilinear form $a$ are presented in the following theorem (Ern and Guermond, 2004).

Theorem 2. Consider the assumptions (11). If u solves (13), then $\mathcal{L}(u)=f$ a.e. in $\Omega$ and $\mathcal{B}(u)=g$ a.e. on $\partial \Omega$.

The bilinear form a is continuous on $H^{1}(\Omega) \times$ $H^{1}(\Omega)$, i.e., a constant $a_{0}>0$ exists such that, for all $u, v \in H^{1}(\Omega)$,

$$
|a(u, v)| \leq a_{0}\|u\|_{H^{1}(\Omega)}\|v\|_{H^{1}(\Omega)} .
$$

Now consider the assumption (12). Let

$$
\begin{aligned}
& \mu_{0}=\underset{x \in \Omega}{\operatorname{essinf}}\left(\mu-\frac{1}{2} \nabla \cdot \beta\right), \quad \gamma_{0}=\underset{x \in \partial \Omega}{\operatorname{essinf}}\left(\gamma+\frac{1}{2} \mathbf{n} \cdot \beta\right) . \\
& \text { If } \mu_{0} \geq 0, \gamma_{0} \geq 0 \text { and } \mu_{0}+\gamma_{0}>0 \text {, then } \\
& \quad \exists \alpha>0, \forall u \in H^{1}(\Omega), \quad a(u, u) \geq \alpha\|u\|_{H^{1}(\Omega)}^{2}, \quad \text { (16) }
\end{aligned}
$$

i.e., a is a coercive bilinear form. Finally the problem (13) admits a unique solution which results from the LaxMilgram lemma.

In summary, considering the hypothesis of Theorem 2, we find a unique solution for the problem 13. which satisfies (3). But $H^{1}(\Omega)$ is an infinite dimensional space and it is not possible to set up a practical method for finding the solution of (13).

Instead, we replace the space $H^{1}(\Omega)$ by a finite dimensional space $V^{n} \subset C^{1}(\bar{\Omega}) \subset H^{1}(\Omega)$,

$$
V^{n}=\operatorname{span}\left\{\Phi_{I}^{n}(X): X \in \Omega, I \in \Lambda^{n}\right\},
$$

where $\Lambda^{n}$ is defined in (10).

Remark 2. The support of some of the basis functions in $V^{n}$ is not a subset of $\Omega$ (but it has intersection with it), hence the basis functions are not orthogonal on $\Omega$. To benefit the orthogonality of wavelets, it is needed to consider a region larger than $\Omega$, which is a motivation of developing fictitious domain methods (see Section 5], but we do not adopt this approach and hence lose orthogonality for some of basis functions. 
Table 1. Scaling coefficients and the Sobolev exponent for Coiflets of degree $N=2,3,4$.

\begin{tabular}{|c|r|r|r|r|r|r|}
\hline$N, \alpha$ & \multicolumn{1}{|c|}{$n$} & \multicolumn{1}{c|}{$a_{n}$} & $n$ & \multicolumn{1}{c|}{$a_{n}$} & \multicolumn{1}{c|}{$n$} & $a_{n}$ \\
\hline \hline & -1 & 0.3293321039 & 0 & 1.0148202640 & 1 & 0.8870036884 \\
$N=2$ & 2 & -0.0444607922 & 3 & -0.2620036884 & 4 & 0.0444607922 \\
$\alpha=1.776$ & 5 & 0.0456678961 & 6 & -0.0148202640 & & \\
\hline & -5 & 0.0520389765 & -4 & -0.0094758721 & -3 & -0.2736020527 \\
$N=3$ & -2 & 0.0217240312 & -1 & 0.8865184464 & 0 & 1.0078625966 \\
$\alpha=2.174$ & 1 & 0.3366672127 & 2 & -0.0591732556 & 3 & 0.0013235641 \\
& 4 & 0.0552419573 & 5 & -0.0029461469 & 6 & -0.0161794573 \\
\hline & -4 & -0.0033518054 & -3 & -0.0233262230 & -2 & 0.0091906207 \\
$N=4$ & -1 & 0.3911561391 & 0 & 1.0043239777 & 1 & 0.8550501499 \\
$\alpha=2.221$ & 2 & -0.0421660096 & 3 & -0.3027375302 & 4 & 0.0589250368 \\
& 5 & 0.0912436455 & 6 & -0.0344902265 & 7 & -0.0102986573 \\
\hline
\end{tabular}

Let $u_{n}, v_{n} \in V^{n}$ and $S^{n} \sigma$ and $S^{n} \beta$ be a matrix and a vector with entries

$$
\left[S^{n} \sigma^{p q}\right], \quad\left[S^{n} \beta^{p}\right], \quad p, q=1, \ldots, d .
$$

We proposed the following approximation of operators $a, l$ in 13):

$$
\begin{aligned}
a_{n}\left(u_{n}, v_{n}\right)= & \int_{\Omega} \nabla v_{n} \cdot S^{n} \sigma \cdot \nabla u_{n}+\int_{\Omega}\left(S^{n} \beta \cdot \nabla u_{n}\right) v_{n} \\
& +\int_{\Omega} S^{n} \mu u_{n} v_{n}+\oint_{\partial \Omega} S^{n} \gamma u_{n} v_{n}, \\
l_{n}\left(v_{n}\right)= & \int_{\Omega} S^{n} f v_{n}+\oint_{\partial \Omega} S^{n} g v_{n} .
\end{aligned}
$$

Hence we considered

$$
\left\{\begin{array}{l}
\text { Find } u_{n} \in V^{n} \subset H^{1}(\Omega), \text { s.t. } \\
a_{n}\left(u_{n}, v_{n}\right)=l_{n}\left(v_{n}\right), \quad \forall v_{n} \in V^{n},
\end{array}\right.
$$

and we aim to prove $\left\|u-u_{n}\right\|_{H^{1}(\Omega)} \leq c 2^{-n N}$, where $u$ solves (3). Since $\left\|u-S^{n} u\right\|_{H^{1}(\Omega)} \leq c 2^{-n N}$, it suffices to obtain

$$
\left\|u_{n}-S^{n} u\right\|_{H^{1}(\Omega)} \leq c 2^{-n N},
$$

and then apply the triangle inequality. We begin with an immediate result of Proposition 1.

Corollary 1. Let $\mathcal{L}$ be an elliptic operator. Then there exists $n \geq 0$ and $\sigma_{0}^{n}>0$ such that, for all $\xi \in \mathbb{R}^{d}$,

$$
\xi \cdot S^{n} \sigma \cdot \xi=\sum_{i, j=1}^{d} S^{n} \sigma_{i j} \xi_{i} \xi_{j} \geq \sigma_{0}^{n}\|\xi\|_{2}^{2} \quad \text { a.e. } i n \Omega .
$$

Moreover, the decreasing sequence $\left\{\sigma_{0}^{n}\right\}$ converges to $\sigma_{0}$.

Applying Proposition 1 and adopting the proof of Theorem 2, we obtain what follows.
Theorem 3. Let

$$
\begin{aligned}
& \mu_{0}^{n}=\underset{x \in \Omega}{\operatorname{essinf}}\left(S^{n} \mu-\frac{1}{2} \nabla \cdot S^{n} \beta\right), \\
& \gamma_{0}^{n}=\underset{x \in \partial \Omega}{\operatorname{essinf}}\left(S^{n} \gamma+\frac{1}{2} \mathbf{n} \cdot S^{n} \beta\right) .
\end{aligned}
$$

(i) There exists $n \geq 0$ such that $\mu_{0}^{n} \geq 0, \gamma_{0}^{n} \geq 0$, and $\mu_{0}^{n}+\gamma_{0}^{n}>0$.

(ii) The sequences $\left\{\mu_{0}^{n}\right\},\left\{\gamma_{0}^{n}\right\}$ converge to $\mu_{0}, \gamma_{0}$, respectively.

(iii) There exists $n \geq 0$ such that $a_{n}$ becomes a coercive operator, i.e., a constant $\alpha_{n}>0$ exists such that

$$
\forall u_{n} \in V^{n}, \quad a_{n}\left(u_{n}, u_{n}\right) \geq \alpha_{n}\left\|u_{n}\right\|_{H^{1}(\Omega)}^{2},
$$

and sequence $\left\{\alpha_{n}\right\}$ converges to $\alpha$.

To obtain (22), we prove the closeness of the operators $a_{n}$ and $a$.

Proposition 2. Let $u \in H^{1}(\Omega)$ and $v_{n} \in V^{n}$. Then

$$
\begin{aligned}
&\left|\int_{\Omega} \nabla v_{n} \cdot S^{n} \sigma \cdot \nabla S^{n} u-\nabla v_{n} \cdot \sigma \cdot \nabla u\right| \\
& \leq c 2^{-n N}\left\|v_{n}\right\|_{H^{1}(\Omega)} \\
&\left|\oint_{\partial \Omega} S^{n} \gamma S^{n} u v_{n}-\gamma u v_{n}\right| \leq c 2^{-n N}\left\|v_{n}\right\|_{H^{1}(\Omega)} .
\end{aligned}
$$

Proof. For $i, j=1, \ldots, d$, the Schwartz inequality yields

$$
\begin{aligned}
& \left|\left\langle\frac{\partial S^{n} u}{\partial x_{i}} S^{n} \sigma_{i j}-\frac{\partial u}{\partial x_{i}} \sigma_{i j}, \frac{\partial v_{n}}{\partial x_{j}}\right\rangle\right| \\
& \quad \leq\left\|v_{n}\right\|_{H^{1}(\Omega)}\left\|\frac{\partial S^{n} u}{\partial x_{i}} S^{n} \sigma_{i j}-\frac{\partial u}{\partial x_{i}} \sigma_{i j}\right\|_{L^{2}(\Omega)} .
\end{aligned}
$$

Since $S^{n} u \in C^{1}(\bar{\Omega})$ (hence its partial derivatives are 
bounded on $\bar{\Omega}$ ), by the triangle inequality we obtain

$$
\begin{aligned}
\| \frac{\partial S^{n} u}{\partial x_{i}} S^{n} \sigma_{i j} & -\frac{\partial u}{\partial x_{i}} \sigma_{i j} \|_{L^{2}(\Omega)} \\
& \leq\left\|\frac{\partial S^{n} u}{\partial x_{i}} S^{n} \sigma_{i j}-\frac{\partial S^{n} u}{\partial x_{i}} \sigma_{i j}\right\|_{L^{2}(\Omega)} \\
& +\left\|\frac{\partial S^{n} u}{\partial x_{i}} \sigma_{i j}-\frac{\partial u}{\partial x_{i}} \sigma_{i j}\right\|_{L^{2}(\Omega)} \\
& \leq\left\|\frac{\partial S^{n} u}{\partial x_{i}}\right\|_{L^{\infty}(\Omega)}\left\|S^{n} \sigma_{i j}-\sigma_{i j}\right\|_{L^{2}(\Omega)} \\
& +\left\|\sigma_{i j}\right\|_{L^{\infty}(\Omega)}\left\|\frac{\partial S^{n} u}{\partial x_{i}}-\frac{\partial u}{\partial x_{i}}\right\|_{L^{2}(\Omega)} \\
& \leq c 2^{-n N}, \quad i, j=1, \ldots, d .
\end{aligned}
$$

Summation on $i$ and $j$ proves 25). To establish 26, apply the trace theorem,

$$
\|v\|_{L^{2}(\partial \Omega)} \leq c\|v\|_{H^{1}(\Omega)},
$$

and substitute $L^{2}(\Omega)$ with $L^{2}(\partial \Omega)$. The same arguments give the desired result.

Considering (25) and (26), it is easy to prove what follows.

Corollary 2. If the contributed functions are smooth enough, then for all $u \in H^{1}(\Omega)$

$$
\begin{aligned}
\left|a_{n}\left(S^{n} u, v_{n}\right)-a\left(u, v_{n}\right)\right| & \leq c 2^{-n N}\left\|v_{n}\right\|_{H^{1}(\Omega)} \\
\left|l_{n}\left(v_{n}\right)-l\left(v_{n}\right)\right| & \leq c 2^{-n N}\left\|v_{n}\right\|_{H^{1}(\Omega)}
\end{aligned}
$$

for a function $v_{n} \in V^{n}$.

Corollary 2 prepare us to state the main result.

Theorem 4. The sequence $u_{n}$ of solutions of (21) converges to $u$, the solution of (3), with the rate of $O\left(2^{-n N}\right)$, i.e.,

$$
\left\|u-u_{n}\right\|_{H^{1}(\Omega)} \leq c 2^{-n N} .
$$

Proof. Since $a_{n}$ is bilinear, the relations (27) and (28) give

$$
\begin{aligned}
a_{n}\left(S^{n} u-u_{n}, v_{n}\right)= & a_{n}\left(S^{n} u, v_{n}\right)-a\left(u, v_{n}\right) \\
& +l\left(v_{n}\right)-l_{n}\left(v_{n}\right) \\
\leq & c 2^{-n N}\left\|v_{n}\right\|_{H^{1}(\Omega)} .
\end{aligned}
$$

Replace $v_{n}$ with $S^{n} u-u_{n} \in V^{n}$ and apply the coercivity of $a_{n}$

$$
\begin{aligned}
\alpha_{n}\left\|S^{n} u-u_{n}\right\|_{H^{1}(\Omega)}^{2} & \leq a_{n}\left(S^{n} u-u_{n}, S^{n} u-u_{n}\right) \\
& \leq c 2^{-n N}\left\|S^{n} u-u_{n}\right\|_{H^{1}(\Omega)} .
\end{aligned}
$$

Thus we obtain (22). Recall that $\left\{\alpha_{n}\right\}$ converges to $\alpha$.

The triangle inequality gives

$$
\begin{aligned}
\left\|u_{n}-u\right\|_{H^{1}(\Omega)} \leq & \left\|u_{n}-S^{n} u\right\|_{H^{1}(\Omega)} \\
& +\left\|S^{n} u-u\right\|_{H^{1}(\Omega)} \leq c 2^{-n N} .
\end{aligned}
$$

Since $u_{n} \in V^{n}$, we let

$$
u_{n}(X):=2^{-\frac{d}{2} n} \sum_{I \in \Lambda^{n}} u_{I}^{n} \Phi_{I}^{n}(X) .
$$

The unknowns $\left\{u_{I}^{n}\right\}_{I \in \Lambda^{n}}$ should be determined from (21) by letting $v_{n}:=\Phi_{J}^{n}, J \in \Lambda^{n}$,

$$
\sum_{I \in \Lambda^{n}} u_{I}^{n} a_{n}\left(\Phi_{I}^{n}, \Phi_{J}^{n}\right)=2^{\frac{d}{2} n} l_{n}\left(\Phi_{J}^{n}\right), \quad J \in \Lambda^{n} .
$$

Equation 30 is a linear system and its matrix is positive definite; see the following theorem, which is proved in any finite element book (e.g. Ern and Guermond, 2004).

Theorem 5. Let $A$ and $b$ be a matrix and a vector, respectively, with entries

$$
A_{J I}:=a_{n}\left(\Phi_{I}^{n}, \Phi_{J}^{n}\right), \quad b_{J}:=2^{\frac{d}{2} n} l_{n}\left(\Phi_{J}^{n}\right),
$$

for $I, J \in \Lambda^{n}$. Then $u_{n}$ in 29) solves (21) iff $U_{n}:=$ $\left\{u_{I}^{n}\right\}_{I \in \Lambda^{n}}$ solves $A x=b$. Since $a_{n}$ is coercive, the stiffness matrix $A$ is positive definite and therefore nonsingular.

\section{Connection coefficients on the interval}

Let $\varphi$ be a Coiflet of degree $N$, with compact support $\left[N_{0}, N_{1}\right]$, and let

$$
N_{2}=N_{1}-N_{0}-1, \quad d_{1}, d_{2} \in\{0,1\}, \quad d_{1}+d_{2} \leq 1 .
$$

For a level of approximation $2^{n} \geq N_{2}$ and for $i, j, k \in \mathbb{Z}$, we want to evaluate the values of

$$
\Gamma_{i j k}^{n, d_{1}, d_{2}}:=\int_{0}^{2^{n}} \varphi_{i}^{\left(d_{1}\right)}(x) \varphi_{j}^{\left(d_{2}\right)}(x) \varphi_{k}^{\left(d_{2}\right)}(x) \mathrm{d} x,
$$

which are called trivariate connection coefficients. These values enhance the precision and speed of the computation for obtaining the linear system. The values of (32) are combinations of ordinary and boundary connection coefficients. The former are defined as follows:

$$
\begin{array}{r}
\Upsilon_{i j}^{d_{1}, d_{2}}:=\int \varphi^{\left(d_{1}\right)}(x) \varphi_{i}^{\left(d_{2}\right)}(x) \varphi_{j}^{\left(d_{2}\right)}(x) \mathrm{d} x, \\
i, j \in \mathbb{Z}, \quad|i|,|j|,|i-j| \leq N_{2} .
\end{array}
$$

For other values of $i, j$, there is no intersection between the support of integrands, so they vanish. Methods for evaluating these values for an arbitrarily wavelet scaling function are proposed in various references (e.g., Latto et $a l ., 1992)$ hence we assume they are definite values.

We define boundary connection coefficients as

$$
\begin{aligned}
\Lambda_{i j k}^{d_{1}, d_{2}} & :=\int_{0}^{\infty} \varphi_{i}^{\left(d_{1}\right)}(x) \varphi_{j}^{\left(d_{2}\right)}(x) \varphi_{k}^{\left(d_{2}\right)}(x) \mathrm{d} x, \\
& |i-j|,|i-k|,|j-k| \leq N_{2}, \quad i, j, k \geq 1-N_{1} .
\end{aligned}
$$


For other values of $i, j, k$, there is no intersection between the support of integrands or/and interval $[0, \infty)$, so they vanish.

If $r=\max (i, j, k) \geq-N_{0}$, then the support of integrand $\varphi_{r}$ in (34), is a subset of $[0, \infty)$, and therefore the bounds of integral in (34) extend to $\mathbb{R}$. In this case a simple change of variation $t=x-i$ gives

$$
\begin{aligned}
\Lambda_{i j k}^{d_{1}, d_{2}} & =\int \varphi^{\left(d_{1}\right)}(t) \varphi_{j-i}^{\left(d_{2}\right)}(t) \varphi_{k-i}^{\left(d_{2}\right)}(t) \mathrm{d} t \\
& =\Upsilon_{j-i, k-i}^{d_{1}, d_{2}},
\end{aligned}
$$

where $\Upsilon_{j-i, k-i}^{d_{1}, d_{2}}$ are known values.

Hence the values of $\Lambda_{i j k}^{d_{1}, d_{2}}$ are unknown only for $i, j, k=1-N_{1}, \ldots,-N_{0}-1$, and to evaluate them, we replace (4) into (34) to obtain a linear system,

$$
\begin{aligned}
\Lambda_{i j k}^{d_{1}, d_{2}} & \int_{0}^{\infty} 2^{d_{1}} \sum_{p} a_{p} \varphi_{2 i+p}^{\left(d_{1}\right)}(2 x) \cdot 2^{d_{2}} \sum_{q} a_{q} \varphi_{2 j+q}^{\left(d_{2}\right)}(2 x) \\
& \cdot 2^{d_{2}} \sum_{r} a_{r} \varphi_{2 k+r}^{\left(d_{2}\right)}(2 x) \mathrm{d} x \\
= & 2^{d_{0}} \sum_{p, q, r} a_{p} a_{q} a_{r} \\
& \int_{0}^{\infty} \varphi_{2 i+p}^{\left(d_{1}\right)}(x) \varphi_{2 j+q}^{\left(d_{2}\right)}(x) \varphi_{2 k+r}^{\left(d_{2}\right)}(x) \mathrm{d} x \\
= & 2^{d_{0}} \sum_{p, q, r=N_{0}}^{N_{1}} a_{p} a_{q} a_{r} \Lambda_{2 i+p, 2 j+q, 2 k+r}^{d_{1}, d_{2}}
\end{aligned}
$$

where $d_{0}=d_{1}+2 d_{2}-1$. A serious problem is that the nonhomogeneous linear system (36) may be singular. By reduction of dependent values we may find nonsingular systems.

1. If $d_{1}=d_{2}=0$, then $\Lambda_{i j k}^{0,0}=\Lambda_{j k i}^{0,0}=\Lambda_{k i j}^{0,0}$, and hence we need to evaluate $\Lambda_{i j k}^{0,0}$ only for $i \geq j \geq k$. The resulting linear system is nonsingular.

2. If $d_{1}=1, d_{2}=0$, then integration by part gives

$$
\begin{aligned}
\Lambda_{i j k}^{1,0}+\Lambda_{j i k}^{1,0}+\Lambda_{k j i}^{1,0} & =-\left(\varphi_{i} \varphi_{j} \varphi_{k}\right)(0), \\
\Lambda_{i i i}^{1,0} & =-\frac{1}{3} \varphi_{i}^{3}(0) .
\end{aligned}
$$

These equations with $\Lambda_{i j k}^{1,0}=\Lambda_{i k j}^{1,0}$ show that we need to evaluate $\Lambda_{i j k}^{1,0}$ only for $i \geq j \geq k$ and $j \geq i \geq k$. The resulting linear system is nonsingular, again.

3. If $d_{1}=0, d_{2}=1$, then $\Lambda_{i j k}^{0,1}=\Lambda_{i k j}^{0,1}$ and we cannot further reduce the unknowns. The resulting linear system is singular and we should add some (not necessarily nonhomogeneous) constraints to obtain the unique solution. Commonly, these constraints are constructed by using moment conditions (Romine and Peyton, 1997; Lin and Zhou, 2001). For arbitrary $i, s$, Eqn. (7) yields

$$
\begin{aligned}
& \varphi_{i}(x) \cdot \sum_{j} j^{s} \varphi_{j}^{\prime}(x) \cdot \sum_{k} \varphi_{k}^{\prime}(x)=0 \\
& \Rightarrow \sum_{j k} j^{s} \Lambda_{i j k}^{0,1}=0 .
\end{aligned}
$$

Now different constraints may be added to settle the rank deficiency.

As yet, we have evaluated (and saved) the values of (33) and (34). To evaluate (32), first we notice that $\Gamma_{i j k}^{n, d_{1}, d_{2}}$ can be nonzero only for

$$
\begin{array}{r}
i, j, k=1-N_{1}, \ldots, 2^{n}-N_{0}-1, \\
|i-j|,|i-k|,|j-k| \leq N_{2} .
\end{array}
$$

Considering $2^{n} \geq N_{2}$, we divide the feasible region (38) into three separate parts:

1. $i, j, k=1-N_{1}, \ldots,-N_{0}-1$. Since the support of $\varphi_{i}^{\left(d_{1}\right)}$ is a subset of $\left[-N_{2}, 2^{n}\right]$, the upper bound of integral in (32) is ineffective. Consequently, $\Gamma_{i j k}^{n, d_{1}, d_{2}}=\Lambda_{i j k}^{d_{1}, \overline{d_{2}}}$

2. $i \vee j \vee k=-N_{0}, \ldots, 2^{n}-N_{1}$. Without loss of generality, consider $-N_{0} \leq i \leq 2^{n}-N_{1}$. Since the support of $\varphi_{i}^{\left(d_{1}\right)}$ is a subset of $\left[0,2^{n}\right]$, the integral bounds of (32) are ineffective and a simple change of variation gives

$$
\Gamma_{i j k}^{n, d_{1}, d_{2}}=\Upsilon_{j-i, k-i}^{d_{1}, d_{2}}
$$

3. $i, j, k=2^{n}-N_{1}+1, \cdots, 2^{n}-N_{0}-1$. A change of variation with $t=x+2^{n}$, gives

$$
\begin{aligned}
& \Gamma_{i-2^{n}, j-2^{n}, k-2^{n}}^{n, d_{1}, d_{2}} \\
& \quad=\int_{2^{n}}^{2^{n+1}} \varphi_{i}^{\left(d_{1}\right)}(x) \varphi_{j}^{\left(d_{2}\right)}(x) \varphi_{k}^{\left(d_{2}\right)}(x) \mathrm{d} x,
\end{aligned}
$$

and therefore

$$
\begin{aligned}
\Gamma_{i j k}^{n, d_{1}, d_{2}} & +\Gamma_{i-2^{n}, j-2^{n}, k-2^{n}}^{n, d_{1}, d_{2}} \\
& =\int_{0}^{2^{n+1}} \varphi_{i}^{\left(d_{1}\right)}(x) \varphi_{j}^{\left(d_{2}\right)}(x) \varphi_{k}^{\left(d_{2}\right)}(x) \mathrm{d} x \\
& =\int \varphi_{i}^{\left(d_{1}\right)}(x) \varphi_{j}^{\left(d_{2}\right)}(x) \varphi_{k}^{\left(d_{2}\right)}(x) \mathrm{d} x \\
& =\Upsilon_{j-i, k-i}^{d_{1}, d_{2}} .
\end{aligned}
$$

Notice that, for $2^{n}-N_{1}+1 \leq i \leq 2^{n}-N_{0}-1$, the support of $\varphi_{i}^{\left(d_{1}\right)}$ is a subset of $\left[0,2^{n+1}\right]$. Moreover, $\left(i-2^{n}, j-2^{n}, k-2^{n}\right)$ lies in Region 1 . Therefore,

$$
\Gamma_{i j k}^{n, d_{1}, d_{2}}=\Upsilon_{j-i, k-i}^{d_{1}, d_{2}}-\Lambda_{i-2^{n}, j-2^{n}, k-2^{n}}^{n, d_{1}, d_{2}} .
$$


We summarize the above discussion in a simple function code. Henceforth $n$ is omitted for simplicity.

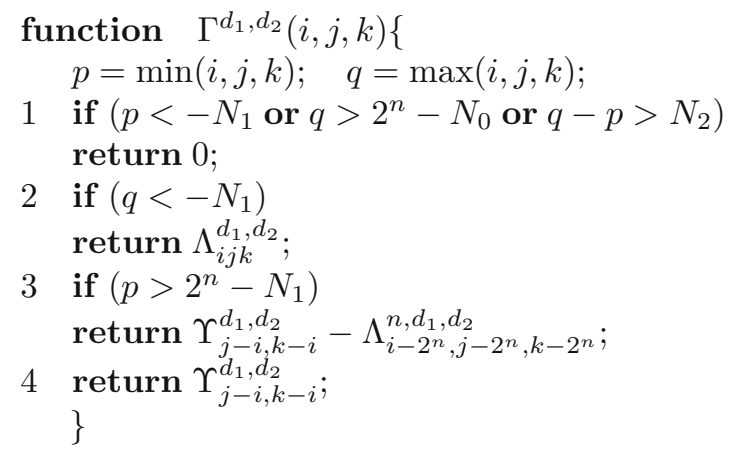

Lines 1 to 4 show that $(i, j, k)$ lies outside of the feasible region (38), inside Region 1, Region 3 and Region 2, respectively. In next section we show that how these values help to construct the linear system (31).

\section{Implementation of the Coiflet-Galerkin method}

The fictitious domain approach is a usual way of treating boundary value problems (Baccou and Liandrat, 2006; Glowinski et al., 2006). In this method, the boundary value problem on $\Omega$ is extended to a linear variational problem involving a parameter $\epsilon$ in a rectangular region, say, $\hat{\Omega}$ containing $\Omega$, involving simple boundary conditions. A solution to the variational problem in $\hat{\Omega}$ restricted to $\bar{\Omega}$ will converge on $\bar{\Omega}$ to a solution of the main boundary value problem, as the penalty parameter $\epsilon$ converges to zero (Resnikoff and Wells, 1998).

The advantage is that the numerical treating of a boundary value problem will be (nearly) independent of the nature of the boundary of $\Omega$, in contrast to many finite element methods whose code must be adapted to the shape of the boundary (Resnikoff and Wells, 1998). The disadvantage is that the computational cost increases, specially when $\Omega$ has a simple shape. We do not adopt this approach, because we want to investigate the solution on $\Omega=(0,1)^{d}$ and our approximation space $V^{n}$ is smaller than the fictitious domain approach.

For simplicity, we let $d=2$ and the result can be used for $d=1,3$ with obvious modifications especially for boundary integrals. Henceforth $\Omega=(0,1)^{2}$. The solution is presented in 29) and should be determined by the linear system (30), which we construct soon. The index set $\Lambda^{n}$ is (see 107)

$$
\Lambda^{n}=\left\{(i, j) \in \mathbb{Z}^{2} \mid i, j=1-N_{0}, \ldots, 2^{n}-N_{1}-1\right\},
$$

and we use the following notation for 30 :

$$
\begin{gathered}
u_{I}^{n}=u_{i j}^{n}, \quad X=\left(x_{1}, x_{2}\right)=(x, y), \\
\Phi_{I}^{n}(X)=\varphi_{n i}(x) \varphi_{n j}(y), \quad \Phi_{J}^{n}(X)=\varphi_{n k}(x) \varphi_{n l}(y) .
\end{gathered}
$$

Frequently, the following three integrals appear in computations:

$$
\begin{aligned}
\int_{0}^{1} \varphi_{n k}(t) \frac{\mathrm{d}}{\mathrm{d} t} \varphi_{n j}(t) \frac{\mathrm{d}}{\mathrm{d} t} \varphi_{n i}(t) \mathrm{d} t & =2^{\frac{5}{2} n} \Gamma_{k j i}^{01} \\
\int_{0}^{1} \varphi_{n k}(t) \varphi_{n i}(t) \frac{\mathrm{d}}{\mathrm{d} t} \varphi_{n j}(t) \mathrm{d} t & =2^{\frac{3}{2} n} \Gamma_{j k i}^{10} \\
\int_{0}^{1} \varphi_{n k}(t) \varphi_{n j}(t) \varphi_{n i}(t) \mathrm{d} t & =2^{\frac{n}{2}} \Gamma_{k j i}^{00}
\end{aligned}
$$

It is obvious that

$$
\begin{aligned}
\frac{\partial}{\partial x} \Phi_{I}^{n} & =\varphi_{n j}(y) \frac{\mathrm{d}}{\mathrm{d} x} \varphi_{n i}(x), \\
\frac{\partial}{\partial y} \Phi_{J}^{n} & =\varphi_{n k}(x) \frac{\mathrm{d}}{\mathrm{d} y} \varphi_{n l}(y) .
\end{aligned}
$$

5.1. Computation of the stiffness matrix. As for (19), the first integral of $a_{n}\left(\Phi_{J}^{n}, \Phi_{I}^{n}\right)$ is

$$
\begin{aligned}
& \int_{\Omega} \nabla \Phi_{J}^{n} \cdot S^{n} \sigma \cdot \nabla \Phi_{I}^{n} \mathrm{~d} X \\
& =\sum_{p, q=1}^{2} \int_{\Omega} \frac{\partial}{\partial x_{q}} \Phi_{J}^{n}(X) S^{n} \sigma^{p q}(X) \frac{\partial}{\partial x_{q}} \Phi_{I}^{n}(X) \mathrm{d} X .
\end{aligned}
$$

Let

$$
\begin{gathered}
S^{n} \sigma^{p q}(x, y)=2^{-n} \sum_{r s} \sigma_{n r s}^{p q} \varphi_{n r}(x) \varphi_{n s}(y), \\
\sigma_{n r s}^{p q}:=\sigma^{p q}\left(\frac{r}{2^{n}}, \frac{s}{2^{n}}\right), \quad p, q=1,2,
\end{gathered}
$$

to obtain

$$
\begin{aligned}
\int_{\Omega} S^{n} \sigma^{11}(X) \frac{\partial}{\partial x} \Phi_{I}^{n}(X) \frac{\partial}{\partial x} \Phi_{J}^{n}(X) \mathrm{d} X \\
=\int_{\Omega} 2^{-n} \sum_{r s} \sigma_{n r s}^{11} \varphi_{n r}(x) \varphi_{n s}(y) \\
\cdot \varphi_{n j}(y) \frac{\mathrm{d}}{\mathrm{d} x} \varphi_{n i}(x) \cdot \varphi_{n l}(y) \frac{\mathrm{d}}{\mathrm{d} x} \varphi_{n k}(x) \mathrm{d} x \mathrm{~d} y \\
=2^{-n} \sum_{r s} \sigma_{n r s}^{11} \int_{0}^{1} \varphi_{n r}(x) \frac{\mathrm{d}}{\mathrm{d} x} \varphi_{n i}(x) \frac{\mathrm{d}}{\mathrm{d} x} \varphi_{n k}(x) \mathrm{d} x \\
\cdot \int_{0}^{1} \varphi_{n s}(y) \varphi_{n j}(y) \varphi_{n l}(y) \mathrm{d} y \\
=2^{2 n} \sum_{r s} \sigma_{n r s}^{11} \Gamma_{r i k}^{01} \Gamma_{s j l}^{00} .
\end{aligned}
$$

Now it is obvious that

$$
\begin{array}{r}
\int_{\Omega} S^{n} \sigma^{21}(X) \frac{\partial}{\partial x} \Phi_{I}^{n}(X) \frac{\partial}{\partial y} \Phi_{J}^{n}(X) \mathrm{d} X \\
=2^{2 n} \sum_{r s} \sigma_{n r s}^{21} \Gamma_{i r k}^{10} \Gamma_{l s j}^{10}
\end{array}
$$




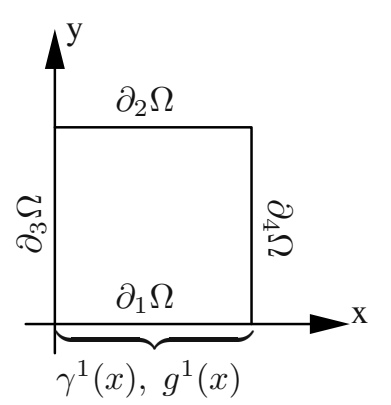

Fig. 1. Boundary condition setting.

Other integrals in (40) are computed similarly. Hence (40) becomes

$$
\begin{aligned}
& \int_{\Omega} \nabla \Phi_{J}^{n} \cdot S^{n} \sigma \cdot \nabla \Phi_{I}^{n} \mathrm{~d} X \\
& =2^{2 n} \sum_{r s}\left(\sigma_{n r s}^{11} \Gamma_{r i k}^{01} \Gamma_{s j l}^{00}+\sigma_{n r s}^{21} \Gamma_{i r k}^{10} \Gamma_{l s j}^{10}\right. \\
& \left.\quad+\sigma_{n r s}^{12} \Gamma_{k i r}^{10} \Gamma_{j l s}^{10}+\sigma_{n r s}^{22} \Gamma_{r i k}^{00} \Gamma_{s j l}^{01}\right) .
\end{aligned}
$$

Remark 3. A wide range of variation of $r, s=1-$ $N_{1}, \ldots, 2^{n}-N_{0}-1$ is not a big problem. Relations of the feasible region (38) imply that, for a fixed test function $v_{n}(X)=\varphi_{n k}(x) \varphi_{n l}(y)$, the index $r$ can vary only in the interval,

$$
\left[\max \left(1-N_{1}, k-N_{2}\right), \min \left(2^{n}-N_{0}-1, k+N_{2}\right)\right] .
$$

Similar results for other indices are obtained.

Now evaluation of other integrals over $\Omega$ in $a_{n}$ is easy,

$$
\begin{aligned}
& \int_{\Omega}\left(S^{n} \beta \cdot \nabla \Phi_{I}^{n}\right) \Phi_{J}^{n} \mathrm{~d} X \\
& =2^{n} \sum_{r s}\left(\beta_{n r s}^{1} \Gamma_{i r k}^{10} \Gamma_{l s j}^{00}+\beta_{n r s}^{2} \Gamma_{i r k}^{00} \Gamma_{j l s}^{10}\right), \\
& \int_{\Omega} S^{n} \mu \Phi_{I}^{n} \Phi_{J}^{n} \mathrm{~d} X=\sum_{r s} \mu_{n r s} \Gamma_{i r k}^{00} \Gamma_{l s j}^{00},
\end{aligned}
$$

where

$$
\begin{aligned}
& \mu_{n r s}:=\mu\left(\frac{r}{2^{n}}, \frac{s}{2^{n}}\right), \\
& \beta_{n r s}^{p}:=\beta^{p}\left(\frac{r}{2^{n}}, \frac{s}{2^{n}}\right), \quad p=1,2 .
\end{aligned}
$$

To treat the boundary integrals in 19 , let $\partial \Omega=$ $\bigcup_{i=1}^{4} \partial_{i} \Omega$, as illustrated in Fig. 1. It is clear that

$$
\oint_{\partial \Omega}=\sum_{i=1}^{4} \oint_{\partial_{i} \Omega} .
$$

On $\partial_{1} \Omega, y=0$ and $0 \leq x \leq 1$. Hence

$$
\begin{aligned}
\oint_{\partial_{1} \Omega} & S^{n} \gamma^{1} \Phi_{I}^{n} \Phi_{J}^{n} \mathrm{~d} s \\
= & \int_{0}^{1} S^{n} \gamma^{1}(x) \Phi_{I}^{n}(x, 0) \Phi_{J}^{n}(x, 0) \mathrm{d} x \\
= & \int_{0}^{1} 2^{-\frac{n}{2}} \sum_{r} \gamma_{n r}^{1} \varphi_{n r}(x) \\
& \cdot \varphi_{n i}(x) \varphi_{n j}(0) \cdot \varphi_{n k}(x) \varphi_{n l}(0) \mathrm{d} x \\
= & 2^{n} \sum_{r} \gamma_{n r}^{1} \zeta_{j s}^{0} \Gamma_{k i r}^{00},
\end{aligned}
$$

where

$$
\zeta_{k l}^{m}:=\varphi_{k}\left(2^{n} m\right) \varphi_{l}\left(2^{n} m\right), \quad \gamma_{n r}^{1}:=\gamma^{1}\left(\frac{r}{2^{n}}\right) .
$$

Hence the boundary integral of $a_{n}$ is

$$
\begin{aligned}
\oint_{\partial \Omega} S^{n} \gamma \Phi_{I}^{n} \Phi_{J}^{n} \mathrm{~d} s & \\
=2^{n} \sum_{r} & \left(\gamma_{n r}^{1} \zeta_{j s}^{0} \Gamma_{k i r}^{00}+\gamma_{n r}^{2} \zeta_{j s}^{1} \Gamma_{k i r}^{00}\right. \\
& \left.\quad+\gamma_{n r}^{3} \zeta_{i k}^{0} \Gamma_{j s l}^{00}+\gamma_{n r}^{4} \zeta_{i k}^{1} \Gamma_{j s l}^{00}\right)
\end{aligned}
$$

Summation of (41)-(44) gives $A_{J I}=a_{n}\left(\Phi_{I}^{n}, \Phi_{J}^{n}\right)$.

5.2. Computation of the right-hand side. First we define

$$
\rho_{i j}=\int_{0}^{2^{n}} \varphi_{i}(x) \varphi_{j}(x) \mathrm{d} x .
$$

A simple way of evaluating (45) is

$$
1=\sum_{k} \varphi_{k}(x) \Rightarrow \rho_{i j}=\sum_{k} \Gamma_{i j k}^{00} .
$$

Now we let

$$
\begin{aligned}
f_{n r s}:=f\left(\frac{r}{2^{n}}, \frac{s}{2^{n}}\right), \quad g_{n r}^{p}:=g^{p}\left(\frac{r}{2^{n}}\right) & \\
p & =1, \ldots, 4,
\end{aligned}
$$

and easily obtain

$$
\begin{aligned}
& \oint_{\Omega}\left(S^{n} f\right) \Phi_{J}^{n} \mathrm{~d} X=2^{-n} \sum_{r s} f_{n r s} \rho_{r k} \rho_{l s}, \\
& \oint_{\partial \Omega}\left(S^{n} g\right) \Phi_{J}^{n} \mathrm{~d} s \\
& =\sum_{r}\left(g_{n r}^{1} \varphi_{s}(0) \rho_{k r}+g_{n r}^{2} \varphi_{s}\left(2^{n}\right) \rho_{k r}\right. \\
& \left.\quad+g_{n r}^{3} \varphi_{r}(0) \rho_{k s}+g_{n r}^{4} \varphi_{r}\left(2^{n}\right) \rho_{k s}\right) .
\end{aligned}
$$

Summation of the two previous equations gives $l_{n}\left(\Phi_{J}^{n}\right)$ and $b_{J}=2^{n} l_{n}\left(\Phi_{J}^{n}\right)$ in 31 . Now we are ready to solve some problems to check the effectiveness of the proposed method. 
Table 2. Relative error norms and the size of linear systems in Example 1.

\begin{tabular}{|c|c|c|c|c|c|c|c|}
\hline$N$ & $n$ & $R E\left(u_{n}\right)_{L^{2}}$ & $R E\left(S^{n} u\right)_{L^{2}}$ & $R E\left(u_{n}\right)_{H^{1}}$ & $R E\left(S^{n} u\right)_{H^{1}}$ & $D A\left(u_{n}\right)_{\infty}$ & size \\
\hline \hline & 5 & $1.1487 e-3$ & $2.6629 e-4$ & $3.7302 e-3$ & $3.7466 e-3$ & $2.2969 e-3$ & 38 \\
2 & 6 & $1.3467 e-4$ & $3.1579 e-5$ & $7.2441 e-4$ & $7.2736 e-4$ & $2.5615 e-4$ & 70 \\
& 7 & $1.6696 e-5$ & $3.9548 e-6$ & $1.0597 e-4$ & $1.0673 e-4$ & $3.1272 e-5$ & 134 \\
\hline & 5 & $1.5956 e-4$ & $1.0358 e-4$ & $3.4494 e-3$ & $3.5158 e-3$ & $4.9805 e-4$ & 42 \\
3 & 6 & $7.6817 e-6$ & $6.0994 e-6$ & $5.7870 e-4$ & $5.8039 e-4$ & $2.0534 e-5$ & 74 \\
& 7 & $4.0643 e-7$ & $3.8998 e-7$ & $6.6928 e-5$ & $6.6937 e-5$ & $1.0612 e-6$ & 138 \\
\hline & 5 & $3.6889 e-2$ & $1.0198 e-5$ & $1.1737 e-2$ & $3.1656 e-4$ & $6.8801 e-2$ & 44 \\
4 & 6 & $7.0988 e-7$ & $2.9025 e-7$ & $2.5253 e-5$ & $2.7234 e-5$ & $1.7135 e-6$ & 76 \\
& 7 & $2.6675 e-8$ & $9.6940 e-9$ & $1.3800 e-6$ & $1.4250 e-6$ & $6.0915 e-8$ & 140 \\
\hline
\end{tabular}

Table 3. Relative error norms and the size of linear systems in Example 2.

\begin{tabular}{|c|c|c|c|c|c|c|c|}
\hline$N$ & $n$ & $R E\left(u_{n}\right)_{L^{2}}$ & $R E\left(S^{n} u\right)_{L^{2}}$ & $R E\left(u_{n}\right)_{H^{1}}$ & $R E\left(S^{n} u\right)_{H^{1}}$ & $D A\left(u_{n}\right)_{\infty}$ & size \\
\hline \hline & 3 & $3.4383 e-3$ & $6.1301 e-4$ & $2.4236 e-2$ & $5.9185 e-3$ & $1.0078 e-2$ & 196 \\
2 & 4 & $2.9695 e-4$ & $7.2015 e-5$ & $3.1318 e-3$ & $9.9353 e-4$ & $9.3381 e-4$ & 483 \\
& 5 & $2.9728 e-5$ & $8.7108 e-6$ & $3.2134 e-4$ & $1.6362 e-4$ & $9.0564 e-5$ & 1444 \\
\hline & 3 & $7.4172 e-4$ & $6.0635 e-4$ & $6.4924 e-3$ & $1.2440 e-2$ & $1.8285 e-3$ & 324 \\
3 & 4 & $5.4123 e-5$ & $3.0341 e-5$ & $\mathbf{9 . 2 4 4 9 e}-\mathbf{4}$ & $1.2492 e-3$ & $1.4012 e-4$ & $\mathbf{6 7 5}$ \\
& 5 & $4.2809 e-6$ & $1.7356 e-6$ & $\mathbf{1 . 2 4 4 8 e}-\mathbf{4}$ & $1.4232 e-4$ & $1.1988 e-5$ & 1763 \\
\hline 4 & 4 & $1.0277 e-5$ & $1.3151 e-6$ & $\mathbf{5 . 0 7 8 6 e}-\mathbf{5}$ & $9.3092 e-5$ & $2.6870 e-5$ & $\mathbf{7 8 3}$ \\
& 5 & $4.1198 e-7$ & $4.9999 e-8$ & $\mathbf{3 . 6 9 0 6 e}-\mathbf{6}$ & $6.6886 e-6$ & $1.1863 e-6$ & 1935 \\
\hline
\end{tabular}

\section{Experimental results}

In this section we present the results of numerical experiments in which we compute solutions of Eqn. (3), subject to the Robin boundary condition. In the previous section we obtained a linear system $A x=b$ for unknowns $U^{n}=\left\{u_{I}^{n}\right\}$ in approximate solution $u_{n}$ in (29). Moreover, $S^{n} u$ in (9), is another approximation of $u$, which we use for comparison study. Because $S^{n} u$ is an approximate function, i.e., considering the exact function $u$ we obtain it, and due to Theorem 1 the high quality of approximation is obvious. We emphasize the $H^{1}$ error norm, because it compares two functions and their behaviour. In the tables we illustrate the following:

1. $R E\left(u_{n}\right)_{L^{2}}$. Relative $L^{2}$ error norm of $u_{n}$; also for $S^{n} u$ in $R E\left(S^{n} u\right)_{L^{2}}$.

2. $R E\left(u_{n}\right)_{H^{1}}$. Relative $H^{1}$ error norm of $u_{n}$; also for $S^{n} u$ in $R E\left(S^{n} u\right)_{H^{1}}$.

3. $D A\left(u_{n}\right)_{\infty}$. Discrete absolute error, i.e., letting $h=$ $1 / 256$, we evaluate

$$
\begin{aligned}
& \max _{i=0, \ldots, 256}\left|u_{n}(i h)-u(i h)\right| \text { in } \mathbb{R} \\
& \max _{i, j=0, \ldots, 256}\left|u_{n}(i h, j h)-u(i h, j h)\right| \text { in } \mathbb{R}^{2} .
\end{aligned}
$$

4. size. Size of vector $U^{n}$.

Example 1. We consider a one dimensional problem,

$$
\left\{\begin{aligned}
-\left(\sigma u^{\prime}\right)^{\prime}+\beta u^{\prime}+\mu u & =f, & & x \in(0,1), \\
\sigma u^{\prime}+\gamma u & =g, & & x=0,1,
\end{aligned}\right.
$$

with data functions,

$$
\begin{aligned}
& \sigma(x)=\frac{1+2 \cos (x)-\ln (\sqrt{1+x})}{1+x^{2}}, \\
& \beta(x)=-\operatorname{atan}(2 x-1)-\mathrm{e}^{\sin (\pi x / 2)}, \\
& \mu(x)=\frac{\cos (x)}{1+2 x}+x-1, \quad \gamma(1)=2, \quad \gamma(0)=1 .
\end{aligned}
$$

Try to find the right-hand side functions $f, g$, if the exact solution is

$$
u(x)=\frac{\mathrm{e}^{-x^{2}}}{\cos (1-x / 2)}-2 x \tanh (x)+\sin (3 \pi x) .
$$

For levels of approximations $n=5,6,7$ and with Coiflets of degrees $N=2,3,4$, we construct and solve the linear system. The condition number of linear systems is of order $O\left(10^{8}\right), O\left(10^{10}\right)$ and $O\left(10^{16}\right)$ for $N=2,3$ and 4 , respectively. Hence the solutions for $N=2,3$ are stable, but instability for $N=4$ needs to apply a regularization technique. We use Matlab routines of Hansen (1994). The results are illustrated in Table 2 and we find that all $H^{1}$ error norms of $u_{n}$ are less than $S^{n} u$, except $N=4, n=5$, where its corresponding condition number is of order $O\left(10^{22}\right)$. Notice the size of linear systems in size.

Example 2. Now we consider a tow-dimensional problem. As we have mentioned, the size of stiffness 
matrix is $\left(2^{n}+N_{2}\right)^{2} \times\left(2^{n}+N_{2}\right)^{2}$, and hence the increment in $n$ considerably enlarges the linear system.

Consider the problem (3) on the domain of Fig. 1 with the following data functions:

$$
\begin{aligned}
\sigma(x, y) & =\left[\begin{array}{cc}
\exp (y-x) & 1+x y \\
2-x y & \cos (x+y)
\end{array}\right] \\
\beta(x, y) & =\left[\begin{array}{c}
x-y \\
y-x
\end{array}\right], \quad \mu(x, y)=\cos (y)+\cosh (x), \\
\gamma_{1}(x) & =1+x, \quad \gamma_{2}=\gamma_{3}=\gamma_{4}=0 .
\end{aligned}
$$

Find the right-hand side functions $f$ and $g$, the exact solution is

$$
u(x, y)=\exp \left(x^{2}-y\right)-\frac{\sin (x)}{\left(1+x+y^{2}\right)} .
$$

For low levels of approximations $n=3,4,5$ and with Coiflets of degrees $N=2,3,4$, we construct and solve the linear system with direct methods or regularization techniques. The condition number of linear systems is of order $O\left(10^{18}\right), O\left(10^{22}\right)$ and $O\left(10^{32}\right)$ for $N=2,3$ and 4 , respectively. The results are illustrated in Table 3 and remarkable values are presented in bold-face. It should be noted that $\|\cdot\|_{H^{1}(\Omega)}$ is a large norm, i.e., it is the summation of the $L^{2}$ norm of a function and all of its partial derivatives.

\section{Conclusion}

With low degree Coiflets $(N=2,3,4)$ and in low scales $(n=3, \ldots, 6)$, we obtained a fine approximation to the exact solution of (3). The size of linear systems was considerably small and we could easily apply regularization techniques for them. The fast rate of convergence helped to keep $n$ small enough, and exact evaluation of trivariate connection coefficients caused to construct linear systems fast and effectively. Experimental results confirmed the proposed approach.

\section{References}

Akbari, H. and Kerayechian, A. (2012). Coiflet-Galerkin method for solving second order BVPs with variable coefficients in three dimensions, Numerical Algorithms 61(4): 681-698, DOI: $10.1007 / \mathrm{s} 11075-012-9558-\mathrm{x}$.

Baccou, J. and Liandrat, J. (2006). Definition and analysis of a wavelet fictitious domain solver for the 2-D heat equation on a general domain, Mathematical Models and Methods in Applied Sciences 16(6): 819-845.

Bandrowski, B., Karczewska, A. and Rozmej, P. (2010). Numerical solutions to integral equations equivalent to differential equations with fractional time, International Journal of Applied Mathematics and Computer Science 20(2): 261-269, DOI: 10.2478/v10006-010-0019-1.
Cerna, D., Finek, V. and Najzar, K. (2008). On the exact values of coefficients of Coiflets, Central European Journal of Mathematics 6(1): 159-169.

Daubechies, I. (1992). Ten Lectures on Wavelets, SIAM, Philadelphia, PA.

El-Gamel, M. (2006). A wavelet-Galerkin method for a singularly perturbed convection-dominated diffusion equation, Applied Mathematics and Computation 181(2): 1635-1644.

Ern, A. and Guermond, J. (2004). Theory and Practice of Finite Elements, Springer, New York, NY.

Glowinski, R., Pan, T.W. and Periaux, J. (2006). Numerical simulation of a multi-store separation phenomenon: A fictitious domain approach, Computer Methods in Applied Mechanics and Engineering 195(41): 5566-5581.

Hansen P.C. (1994). Regularization Tools: A Matlab package for analysis and solution of discrete Ill-posed problems, Numerical Algorithms 6: 1-35, http://www.mathworks.com/matlabcentral/ fileexchange/52

Hashish, H., Behiry, S.H., Elsaid, A. (2009). Solving the 2-D heat equations using wavelet-Galerkin method with variable time step, Applied Mathematics and Computation 213(1): 209-215.

Jensen, T.K. and Hansen, P.C. (2007). Iterative regularization with minimum-residual methods, BIT Numerical Mathematics 47(1): 103-120.

Latto, A., Resnikoff, H. and Tenenbaum, E. (1992). The evaluation of connection coefficients of compactly supported wavele, Proceedings of the Workshop on Wavelets and Turbulence, Princeton, NJ, USA, pp. 76-89.

Lin, E. and Zhou, X. (2001). Connection coefficients on an interval and wavelet solution of Burgers equation, Journal of Computational and Applied Mathematics 135(1): 63-78.

Lin, E.and Zhou, X. (1997). Coiflet interpolation and approximate solutions of partial differential equations, $\mathrm{Nu}$ merical Methods for Partial Differential Equations 13(4): 303-320

Nowak, Ł.D., Pasławska-Południak, M. and Twardowska, K. (2010). On the convergence of the wavelet-Galerkin method for nonlinear filtering, International Journal of Applied Mathematics and Computer Science 20(1): 93-108, DOI: $10.2478 / \mathrm{v} 10006-010-0007-5$.

Reddy, J. (2006). An Introduction to the Finite Element Method, 3rd Edn., McGraw Hill, New York, NY.

Resnikoff, H. and Wells, R.O. Jr (1998). Wavelet Analysis: The Scalable Structure of Information, Springer-Verlag, New York, NY.

Romine, C.H. and Peyton, B.W. (1997). Computing connection coefficients of compactly supported wavelets on bounded intervals, Technical Report ORNL/TM-13413, Computer Science and Mathematical Division, Mathematical Sciences Section, Oak Ridge National Laboratory, Oak Ridge, TN, http://citeseer.ist.psu.edu/ romine97computing.html 
Saad, Y. (1996). Iterative Methods for Sparse Linear Systems, PWS Publishing Company.

Saberi-Nadjafi, J., Mehrabinezhad, M. and Akbari, H. (2012). Solving Volterra integral equations of the second kind by wavelet-Galerkin scheme, Computers and Mathematics with Application 63(11): 1536-1547, DOI: 10.1016/j.camwa.2012.03.043.

Vampa, V., Martin, M. and Serrano, E. (2010). A hybrid method using wavelets for the numerical solution of boundary value problems on the interval, Applied Mathematics and Computation 217(7): 3355-3367.
Hani Akbari is currently a post doctoral researcher at the School of Energy Resources, University of Wyoming, US. He received his Ph.D. in numerical analysis from the Ferdowsi University of Mashhad, Iran. His research field is scientific computation for large scale problems of uncertainly quantification and numerical solution of partial differential equations.
Received: 24 February 2012

Revised: 11 June 2012 